\title{
Spontaneous alternation and learning in rats
}

\author{
DAVID LESTER \\ WELLESLEY COLLEGE
}

Rats that reached the criteria for learning a simple place response in a T-maze sooner showed significantly more spontaneous alternation than slower learning rats in a pre-learning test of exploratory tendencies. The stricter the criterion used for the learning task, the stronger was this association.

If a rat is rewarded in a $\mathrm{T}$-maze for choosing, say, to turn left at the choice point on its first exposure to the maze, then that rat shows a strong tendency to turn right on its next exposure to the maze. Walker (1958) suggested that this conflict between spontaneous alternation and learning could be resolved by separating the two phenomena temporally. Immediately after making a response, regardless of whether the animal is rewarded or not, there is a temporary action decrement phase during which repetition of that response is less likely than it had previously been. If the animal was rewarded for a particular response, this response becomes more likely subsequently, after the action decrement has dissipated, due to the increase in habit strength.

Walker argued that the greater the action decrement after a response, the greater should be the subsequent increment to habit strength in a rewarded animal. Walker \& Paradise (1958) tested different groups of rats in different mazes and found that maze conditions that led to large amounts of spontaneous alternation also led to quicker learning.

A stronger test of Walker's hypothesis would be to use the same rats for both tests and to investigate whether individual differences in spontaneous alternation behavior and speed of learning are associated. The present experiment was designed to this end. Method

Thirty-two male rats of the Charles River Breeding Laboratories, strain $C D$, aged 142-146 days, were used. A Y-maze and a T-maze were each constructed from three pieces of runway, each 22 in. $x 6$ in. $x$ 6 in. high, and made of wood. The mazes were covered with hardware cloth.

The rats were reduced to and maintained at $84 \%$ $86 \%$ body weight. This took 10 days. Then each rat was given one $15 \mathrm{~min}$ exploration experience in the Y-maze and the sequence of arms entered by each rat was noted. An entry was defined as made when all four paws of the rat had entered into an arm.

On Days 14-17, each rat was placed once each day into the goal box of an arm of the T-maze for $5 \mathrm{~min}$, with eight $45-\mathrm{mg}$ Noyes pellets present in a small food cup. The rats experienced the goal boxes either in the order LRLR or RLRL over the four days (where $L$ is the left arm and $R$ is the right arm). Seven rats did not eat the food on these days and were discarded-Group RE (refused to eat).
On Days 18-31, each rat was given four trials a day in the $T$-maze. Half were rewarded in the left arm and half in the right arm. The reward was eight Noyes pellets. The rats were given $2 \mathrm{~min}$ to enter the correct arm, after which they were placed in it. After the rats had entered the correct arm a guillotine door at the entrance to the arm was closed behind them. The rats were given up to 2 min to eat the pellets. The rats were placed in a cage for 15 sec between trials.

After seven days of training, seven rats still refused to enter the correct arm (or either arm) in the 2 min allowed. These animals were discarded -Group RC (refused to choose).

Thus there were three groups of rats: $7 \mathrm{RE}$ (refused to eat), $7 \mathrm{RC}$ (refused to choose), and $18 \mathrm{~L}$ (learners).

\section{Results}

Groups RE, RC, and $\mathrm{L}$ did not differ significantly in the number of arms entered during the exploratory test on a one-way analysis of variance $(F=1.93$, $\mathrm{df}=2 / 29, \mathrm{~ns}) .1$ The mean number of arms entered was: RE, 27.3; RC, 25.4; and L, 31.8.2 The groups did not differ in the amount of alternation during their exploration ${ }^{3}$ on a Kruskal-Wallis one-way anal$y$ sis of variance $(\mathrm{H}=3.89, \mathrm{df}=2, \mathrm{~ns})$. The median percentages of alternation were: RE, $66.7 \%$; $R C$, $59.1 \%$; and $\mathrm{L}, 61.7 \% .4$

The criterion for learning the simple $T$-maze habit was expressed in terms of the number of successive days on which the animal chose correctly on four out of four trials. The longest sequence of successive days on which this criterion was met was correlated with the activity and alternation scores of the animals on the exploration test by means of a Spearman correlation coefficient. The correlation of the longest sequence of criterion days with activity was -0.21 ( $n s)$, and with alternation was $0.46(p<$ 0.05). A total of four rats reached a criterion of four successive days with all choices correct, a total of six reached a criterion of three successive days, and a total of nine reached a criterion of two successive days. The activity and alternation scores of these animals are shown in Table 1, where it can be seen that the rats reaching the criterion showed significantly more alternation in the exploration test than those failing to meet the criterion, especially when very strict criteria are imposed (that is, a longer sequence of successive days with all choices correct). No significant differences in activity scores were found between rats that met the learning criteria and those that failed to meet the criteria. 5 It can be concluded, therefore, that the better 
Table 1. Median exploration scores of rats meeting and failing to meet the leaming criteria. ${ }^{a}$

(a) activity (arms entered)

\begin{tabular}{lccc} 
& $\begin{array}{c}\text { meeting } \\
\text { criterio }\end{array}$ & $\begin{array}{c}\text { not meeting } \\
\text { criterio }\end{array}$ & $\begin{array}{c}\text { Mann-Whitney } \\
\text { U Test }\end{array}$ \\
\hline 4 successive days & $31 \frac{1}{2}$ & 34 & $\mathrm{U}=22, \mathrm{~ns}$ \\
3 successive days & 31 & $351 / 2$ & $\mathrm{U}=20, \mathrm{~ns}$ \\
2 successive days & 32 & 36 & $\mathrm{U}=281 / 2, \mathrm{~ns}$ \\
\hline
\end{tabular}

(b) alternation

\begin{tabular}{lccc} 
& $\begin{array}{c}\text { meeting } \\
\text { criteria }\end{array}$ & $\begin{array}{c}\text { not meeting } \\
\text { criterio }\end{array}$ & $\begin{array}{c}\text { Mann-Whitney } \\
\text { U Test }\end{array}$ \\
\hline 4 successive days & $73.4 \%$ & $59.7 \%$ & $\mathrm{U}=4, \mathrm{p}<0.01$ \\
3 successive days & $72.5 \%$ & $58.7 \%$ & $\mathrm{U}=4, \mathrm{p}<0.001$ \\
2 successive days & $64.9 \%$ & $60.0 \%$ & $\mathrm{U}=26, \mathrm{~ns}$ \\
\hline
\end{tabular}

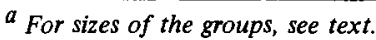

learners of the $\mathrm{T}$-maze habit showed more alternation behavior in the $\mathrm{Y}$-maze test of exploration.

To check upon this result, the proportion of correct choices made by the nine rats with the highest alternation scores on the exploration test was compared with the proportion of correct choices made by the nine rats with the lowest alternation scores. On each of the last eight days of training, those rats with higher alternation scores made more correct choices than the rats with lower alternation scores (binomial $\mathrm{p}=0.004$ ).

\section{Discussion}

This study has confirmed the prediction that rats that show high alternation tendencies will also perform better on a learning task. The learning task used was one in which alternation tendencies would lead to a rat failing to meet the criteria used, namely all choices in a $T$-maze to one arm. The higher alternation tendencies of the good learners does not impede their learning.

An obvious mediating factor in such an association is fear or anxiety. Rats with a high level of fear may be both poor learners and poor explorers. That this is unlikely here is suggested by the fact that the most scared rats (those who refused to eat the food or make a choice in the maze) did not differ from the rats that did learn in their alternation scores on the exploration test. In addition to this, Lester (1967a) has noted that increased anxiety may not necessarily inhibit spontaneous alternation.

The present result may be viewed as allied to the report by Lester (1967b) that dominant rats showed higher alternation tendencies than submissive rats and to a study by Beach (1939) in which good learners were found to be better mothers (if female) and better copulators (if male) than poor learners. It appears that rats who behave well or efficiently in one sphere of behavior behave efficiently in other spheres as well. Whether the mediating factor in this phenomenon is anxiety or some other factor is a problem for future research.

\section{References}

BEACH, F. A. The neural basis of innate behavior: III. Comparison of learning ability and instinctive ability in the rat. J. comp. Psychol, $1939,28,225-268$.

LESTER, D. Sex differences in exploration: Toward a theory of exploration. Psychol. Rec., 1967a, 17, 55-62.

LESTER, D. Exploratory behavior of dominant and submissive rats. Psychon. Sci., 1967b, 9,

MONTGOMERY, K. C. The relation between fear induced by novel stimulation and exploratory drive. J. comp. physiol. Psychol., 1955, 48,254260 .

SIEGEL, S. Nonparametric statistics for the behavioral sciences. New York: McGraw-Hill, 1956.

WALKER, E. L. Action decrement and its relation to learning. Psychol. Rev., 1958, 65, 129-142.

WALKER, E. L., \& PARADISE, N. A positive correlation between action decrement and learning. $J$. exp. Psychol., 1958, 56, 45-47.

\section{Notes}

1. ns indicates $p>0.10$. All tests on activity measures were twotailed tests (since there was no prediction made) whereas all tests on alternation measures were one-tailed tests.

2. The difference between Group $L$ and Groups $R E$ and $R C$ combined approached significance on a $t$ test $(t=1.94, d f=30, p<0.10)$.

3. The method of assessing alternation in Y-maze exploration was that used by Montgomery (1955) and has been described previously (Lester, 1967b).

4. Group $L$ did not differ significantly from Groups $R E$ and $R C$ combined on a Mann-Whitney $U$ Test $(U=1181 \%$, ns). Siegel (1956) was used as the reference for nonparametric tests.

5. For Group $\mathrm{L}$ the Spearman correlation coefficient between activity scores and alternation scores on the exploration test was -0.22 (ns). 